\title{
Transglutaminase 2 overexpression induces depressive-like behavior and impaired TrkB signaling in mice
}

\author{
Chirayu D Pandya ${ }^{1}$, Nasrul Hoda ${ }^{2}$, Amanda Crider $^{1}$, Diya Peter ${ }^{1}$, Ammar Kutiyanawalla $^{1}$, \\ Sanjiv Kumar ${ }^{3}$, Anthony O Ahmed ${ }^{4}$, Gustavo Turecki ${ }^{5}$, Caterina M Hernandez ${ }^{6}$, Alvin V \\ Terry $\mathrm{Jr}^{6}$, and Anilkumar Pillai ${ }^{1}$ \\ ${ }^{1}$ Department of Psychiatry and Health Behavior, Augusta University, Augusta, Georgia, USA \\ ${ }^{2}$ Department of Medical Laboratory, Imaging and Radiologic Sciences, Augusta University, \\ Augusta, Georgia, USA \\ ${ }^{3}$ Vascular Biology Center, Augusta University, Augusta, Georgia, USA \\ ${ }^{4}$ Department of Psychiatry, Weill Cornell Medical College, White Plains, NY, USA \\ ${ }^{5}$ McGill Group for Suicide Studies, Depressive Disorders Program, Douglas Mental Health \\ University Institute, McGill University, Montreal, Quebec, Canada \\ ${ }^{6}$ Department of Pharmacology and Toxicology, Augusta University, Augusta, Georgia, USA
}

\section{Abstract}

Serotonin (5-HT) and brain derived neurotrophic factor (BDNF) are two signaling molecules that play important regulatory roles in the development and plasticity of neural circuits that are known to be altered in depression. However, the mechanism by which 5-HT regulates BDNF signaling is unknown. In the present study, we found that 5-HT treatment increases BDNF receptor, TrkB (tropomyosin related kinase B) levels in mouse primary cortical neurons via a Rac1 (RAS-related C3 botulinum toxin substrate 1)-dependent mechanism. Significant increases in the levels of transglutaminase 2 (TG2, which is implicated in transamidation of 5-HT to Rac1) are observed in the mouse prefrontal cortex (PFC) following chronic exposure to stress. We also found that TG2 levels are increased in the postmortem PFC of depressed suicide subjects relative to matched controls. Moreover, in mice, neuronal overexpression of TG2 resulted in the atrophy of neurons and reduced levels of TrkB in the PFC as well as a depressive-like phenotype. Overexpression of TG2 in mouse cortical neurons reduced TrkB levels as a result of impaired endocytosis of TrkB. TG2 inhibition by either a viral particle or pharmacological approach attenuated behavioral deficits caused by chronic unpredictable stress. Moreover, the overexpression of TrkB in the

Users may view, print, copy, and download text and data-mine the content in such documents, for the purposes of academic research, subject always to the full Conditions of use: http://www.nature.com/authors/editorial_policies/license.html\#terms

Correspondence to: Anilkumar Pillai.

Authors' contributions: A.P contributed to study design, data collection, analysis and writing. C.D.P contributed to data collection, analysis, and writing. A.C., D.P. and A.K. contributed to data collection. N.H and S.K. contributed to lentiviral experiments. A.O.A contributed to statistical analysis. G.T provided the human postmortem tissues. A.T and C.M.H contributed to the animal behavior experiments.

Biomedical Financial Interests and Potential Conflicts of Interest: All authors report no relevant biomedical financial interests or potential conflicts of interest.

Supplementary information is available at Molecular Psychiatry's website. 
mouse PFC ameliorated the depressive-like phenotype of TG2 overexpressed mice. Taken together, these postmortem and preclinical findings identify TG2 as a critical mediator of the altered TrkB expression and depressive-like behaviors associated with chronic exposure to stress and suggest that TG2 may represent a novel therapeutic target in depression.

\section{Introduction}

Major depression is one of the most prevalent and debilitating illnesses worldwide causing an enormous personal and economic burden. Although the therapeutic options for this disorder have been improved over time, it is sobering that depression is still characterized by persistent functional impairments for most patients. Chronic stress is known to contribute to the pathophysiology of depression, and exposure to chronic or repeated stress leads to synaptic changes and depressive -like behaviors in rodents. Moreover, chronic stressinduced depressive behaviors are strongly linked to alterations in serotonin (5hydroxytryptamine, 5-HT) and brain-derived neurotrophic factor (BDNF), two signaling molecules that play regulatory roles in many neuronal functions including neurogenesis, behavior and synaptic plasticity. In addition, a large body of evidence indicates alterations in 5-HT and BDNF signaling pathways in the brain of depressed subjects. Studies also suggest that serotonergic transmission exerts powerful control over BDNF signaling, and enhanced serotonergic transmission may be a key mechanism underlying the therapeutic effects of antidepressants ${ }^{1}$. However, the mechanisms underlying the interactions between 5-HT and BDNF signaling pathways and their relevance to depression are poorly understood.

Recent studies have shown the transamidation of 5-HT to small GTPases such as RhoA and Rac1, via a process catalyzed by enzymes called transglutaminases (TGs) ${ }^{2,3}$. TGs are a family of calcium $(\mathrm{Ca} 2+)$ dependent enzymes that catalyze the formation of intra- and intermolecular bonds between the $\gamma$-carboxyamide moiety of glutamine and primary amino groups ${ }^{4}$. Type-2 transglutaminase (TG2), the most ubiquitous TG isoform, is involved in neural development and functioning 5 . A number of studies have shown that transamidated small GTPases like Rac1 and RhoA are constitutively active and that they undergo rapid proteasomal degradation ${ }^{2,3,6}$. Therefore, increased transamidation results in less 5-HT availability for downstream signaling, a process that has been implicated in the pathophysiology of depression. In the current study, we found that TG2 levels are increased in the prefrontal cortex (PFC) of both chronic stress-exposed mice as well as of depressed suicide subjects. TG2 overexpression in mice resulted in the atrophy of neurons and reduced levels of BDNF receptor, TrkB (tropomyosin related kinase B) in the PFC as well as a depressive-like phenotype. Moreover, overexpression of TrkB in the mouse PFC ameliorated the depressive-like phenotype of TG2 overexpressed mice.

\section{Results}

\section{Rac1 is involved in TrkB regulation and depressive-like behavior}

To determine the effect of 5-HT on TrkB signaling, we first performed in vitro studies in primary cortical neurons. Immunoblot analysis showed a dose and time-dependent effect of 5-HT on TrkB, where 5-HT significantly increased TrkB protein levels at $14 \mu \mathrm{M}$ up to $48 \mathrm{~h}$ 
$(P<0.05)$ (Fig. 1a, b). To examine the possibility that Rac1 could influence the expression of TrkB, we performed in vitro transfection assays in primary cortical neurons. Rac1 pulldown experiments showed that constitutively active Rac1 constructs (CA-Rac1) had more active GTPases than control constructs $(P<0.05)$ (Fig. 1c). TrkB protein levels were significantly higher in cells transfected with CA-Rac1 as compared to WT $(P<0.05)$ (Fig. 1c). Next, we examined whether pharmacological inhibition of Rac1 activity could influence the 5-HT-induced increase in TrkB protein levels. Pre-treatment with NSC 23766, a Rac1 inhibitor attenuated the 5-HT-stimulated increase in TrkB protein levels in neurons $(P<$ 0.05) (Fig. 1d).

We next performed in vivo experiments. Mice administered NSC 23766 for two weeks showed depressive-like behaviors (i.e., increases in the time spent immobile) in the tail suspension test $(P<0.05)$ (Supplementary Fig. 1a) and the forced swim test $(P<0.05)$ (Supplementary Fig. 1b). No significant difference in total distance travelled, distance in the center, or time spent in the periphery or center of an open field was found between vehicle and NSC 23766-treated mice (Supplementary Fig. 1c), indicating that our conclusions about depressive-like behaviors were not confounded by decreases in motor function/locomotor activity or elevated anxiety-like behaviors. NSC 23766 treatment significantly inhibited phosphorylation of TrkB in the mouse PFC $(P<0.05)$ (Supplementary Fig. 1d,e). Furthermore, decreases in the levels of the phosphorylation of Akt $(P<0.05)$ (Supplementary Fig. 1d, f) and Erk $(P<0.05)$ (Supplementary Fig. 1d, g) (i.e., two molecules that are activated in TrkB-mediated pathways) were found in the PFC of mice following Rac1 inhibition.

\section{TG2 overexpression induces reductions in TrkB and Rac1 protein levels in mouse PFC}

Serotonin has been shown to transamidate Rac1 leading to its proteasomal degradation ${ }^{2}$. To understand the mechanism linking serotonin to TrkB, we used mice overexpressing TG2 (hereafter called TG2 mice), the enzyme responsible for the above transmidation process. It has been shown that TG2 is expressed predominantly in the brain of TG2 mice ${ }^{7}$. We found that TG2 overexpression in male mice resulted in higher TG2 protein expression in the PFC, hippocampus and striatum (Fig. 2a). We performed immunofluorescence studies in cortical sections from TG2 mice to examine the cellular localization of TG2. Laser confocal imaging showed that TG2 is selectively colocalized with CamK II+ cells (Supplementary Fig. 2a), not with PV+ cells (Supplementary Fig. 2b). Studies using vGlut1 antibody further confirmed the localization of TG2 in pyramidal neurons (Supplementary Fig. 2c). Rac1 protein levels were significantly lower in the PFC of male TG2 mice $(P<0.05)$ (Fig. 2b) as compared to wildtype littermate controls. We did not find any significant change in Rac1 protein levels in the hippocampus (Supplementary Fig. 3a) and striatum (Supplementary Fig. $3 b)$ of TG2 mice, indicating cortex-specific changes in Rac1 expression in TG2 mice. Also, TG2 overexpression did not cause any change in the levels of RhoA (another small GTPase) in any of the brain regions studied (Supplementary Fig. 3c-e). Since we found a significant reduction in Rac1 protein levels in PFC of TG2 mice and Rac1 is known to be involved in the endocytosis of $\mathrm{TrkB}^{8}$, we next examined TrkB protein levels in WT and TG2 mice. TG2 overexpression reduced the levels of both TrkB $(P<0.05)$ (Fig. $2 \mathrm{c})$ and its phosphorylated form (pTrkB) $(P<0.05)$ (Fig. 2d) in the mouse PFC, but no significant change was observed 
in hippocampus (Supplementary Fig. 3f) or striatum (Supplementary Fig. 3g). In addition, we found significant decreases in the phosphorylation of Akt (pAkt) $(P<0.05)$ (Fig. 2e) and Erk (pErk) $(P<0.05)$ (Fig. 2f) in the PFC of TG2 mice. Because recent studies have implicated other neurotrophic factors such as NGF and VEGF in neuropsychiatric disorders, we next examined the levels of NGF receptor, TrkA and VEGF receptor, Flk1 in WT and TG2 mice. No significant change was found in the levels of TrkA (Supplementary Fig. 3h) and Flk1 (Supplementary Fig. 3i) in PFC of TG2 mice as compared to WT. To determine whether TG2 overexpression in mice causes any change in spine density, Golgi staining was performed. Spines were counted on secondary and tertiary branches of apical dendrites in the superficial layer and deep layers. As shown in Fig. 2g, spine density was reduced in layer $\mathrm{V}$ of PFC in TG2 mice, compared with control littermates.

\section{TG2 overexpression induces depressive-like behavior in mice}

Next, we performed behavioral assays related to schizophrenia, anxiety and depression in WT and TG2 mice. In the Prepulse inhibition (PPI) test (a sensorimotor gating assessment), we used a combination of auditory-evoked startle $(120 \mathrm{~dB})$ and three prepulse levels $(75,80$, and $85 \mathrm{~dB}$ ). PPI scores were analyzed using two-way ANOVA with prepulse intensities as a within-subject factor, and genotype as between-subject factor. We found a significant main effect of prepulse intensity $[\mathrm{F}(2,36)=28.39 ; \mathrm{p}<0.0001]$ and genotype $[\mathrm{F}(1,36)=6.578$; $\mathrm{p}=0.0146]$, however, the genotype $\times$ prepulse intensity interaction was not significant. Posthoc analyses indicated that the \%PPI increased with increasing prepulse intensity ( $<<0.05$ for the $75 \mathrm{~dB}$ and $80 \mathrm{~dB}$ level versus the $70 \mathrm{~dB}$ level) in both WT and TG2 overexpressed mice. While the \%PPI at the $75 \mathrm{~dB}$ level was slightly higher in the TG2 overexpressed mice as compared to WT mice, there were no major differences in PPI between the genotypes (Supplementary Fig. 4a). Data on startle amplitude showed no significant difference between WT and TG2 mice (Supplementary Fig. 4a). There were also no significant differences in spatial alternation in the Y-maze between TG2 and WT mice (Supplementary Fig. 4b) indicating that spatial working memory performance was similar in the two genotypes . Interestingly, we did observe fewer arm entries in the TG2 mice, and visual observation of the videos indicated a considerable amount of stretch and attend behaviors in these mice, potentially indicative of an anxiety-like phenotype. However, more rigorous evaluations of anxiety-like behaviors in the Elevated Plus maze test (Supplementary Fig. 4c), Light/Dark test (Supplementary Fig. 4d) and Open Field (Fig. 2h) did not reveal any significant differences between TG2 and WT mice. We did, however, observe larger distances travelled in the open field chamber suggestive of hyperactivity in TG2 mice as compared to WT mice $(P<0.05)$ (Fig. 2h). TG2 and WT mice were also tested in the tail suspension test (TST) and forced swim test (FST) for depressive-like behaviors. TG2 mice were immobile for a longer period of time than WT mice in the TST $(P<0.05)$ and FST $(P<0.05)$ indicating a depressive-like phenotype in these mice (Fig. 2h). Moreover, TG2 overexpression resulted in a significant decrease in sucrose preference, suggestive of an anhedonia-like phenotype in these mice (Fig. 2h).

To determine whether TG2 overexpression results in alterations in 5-HT levels, we examined 5-HT levels in PFC and hippocampus of WT and TG2 mice. No significant change in 5-HT levels was found in the above brain regions (Supplementary Fig. 5a,b). However, 
significantly higher 5-HT levels were observed in whole blood of TG2 mice as compared to the WT mice $(P<0.05)$ (Fig. 2i). Furthermore, acute injection of the serotonin reuptake inhibitor (antidepressant), fluoxetine $(20 \mathrm{mg} / \mathrm{kg})$ significantly attenuated the hyperactivity in TG2 mice $(P<0.05)$ (Supplementary Fig. 6a). Moreover, fluoxetine significantly increased mobility of TG2 mice in the TST $(P<0.05)$ (Supplementary Fig. 6b) and FST $(P<0.05)$ (Supplementary Fig. 6c). We found that fluoxetine administration also increased TrkB phosphorylation in the PFC in both WT and TG2 mice $(P<0.05)$ (Supplementary Fig. 6d).

\section{PFC-specific overexpression of TG2 induces depressive-like behavior in mice}

Since the changes in Rac1 and TrkB were specific to the PFC of TG2 mice, we investigated whether PFC-specific overexpression of TG2 induces a depressive-like phenotype in mice (Fig. 2j). After stereotaxic lentiviral TG2 gene delivery to the PFC, TG2-GFP was detected in the PFC by immunofluorescence imaging and TG2 immunohistochemistry (Fig. 2k). TG2 protein levels were higher in the PFC samples from lentiviral TG2-injected mice as compared to lentiviral-GFP injected control mice $(P<0.05)$ (Fig. 2l). Moreover, TG2 overexpression induced a significant reduction in TrkB protein levels in the PFC $(P<0.05)$ (Fig. 21). Behavioral analysis at 2 weeks after lentiviral transduction showed that lentiviral TG2-injected mice exhibited hyperactivity in the open field test $(P<0.05)$; and depressivelike behaviors in the TST $(P<0.05)$ and FST $(P<0.05)$ (Fig. $2 \mathrm{~m})$.

\section{Glucocorticoid receptor mediates stress-induced increase in TG2 levels}

Since changes in hypothalamic-pituitary-adrenal (HPA) axis and therefore, glucocorticoid levels are implicated in stress pathophysiology, we examined whether TG2 levels are affected in mice following chronic glucocorticoid exposure. Chronic corticosterone treatment has been shown to induce a depressive-like phenotype and a decrease in TrkB protein levels in mouse $\mathrm{PFC}^{9}$. We did not find any significant change in fluid consumption rate in mice following corticosterone administration for 7 weeks (Supplementary Fig. 7). A significant increase in TG2 protein levels was observed in the PFC of mice following corticosterone exposure $(P<0.05)$ (Supplementary Fig. 8a). In addition, corticosteroneinduced increase in TG2 levels was inhibited by RU486, a glucocorticoid receptor antagonist in primary cortical neurons suggesting that glucocorticoid signaling is involved in the increase in TG2 levels following stress $(P<0.05)$ (Supplementary Fig. 8b).

\section{TG2 mediates the CUS-induced changes in depressive-like behavior and TrkB protein levels in mice}

Next, we examined whether TG2 is involved in chronic stress-induced depressive-like behavior in mice. We used a chronic unpredictable stress (CUS) procedure, which is a well characterized (and predictive) paradigm for producing depressive-like signs in rodents. We found a significant increase in TG2 protein levels in the PFC of mice exposed to CUS for 21 days $(P<0.05)$ (Fig. 3a). To determine the direct role of TG2 in CUS-induced depressivelike behavior, we silenced TG2 expression in mouse PFC using lentiviral vectors expressing TG2 siRNA (Fig. 3b). A significant reduction in TG2 protein levels was observed in mouse PFC following TG2 siRNA administration (Fig. 3c). We found that CUS induced depressive-like behavior (i.e., increased immobility time in TST and FST) in WT mice, but not in TG2 siRNA-injected mice $(P<0.05)$ (Fig. 3d). Moreover, CUS exposure resulted in 
decreased TrkB protein levels in the PFC of WT mice, but not in TG2 siRNA-injected mice (Fig. 3e). The idea that TG2 is responsible for the behavioral effects of CUS was further confirmed using cysteamine, a pharmacological inhibitor of TG2 (Fig. 3f). Cysteamine treatment for 21 days significantly attenuated the CUS-induced increase in immobility time in TST $(P<0.05)$ and FST $(P<0.05)$ in mice (Fig. 3g). No significant difference was observed in open field activity in mice following CUS $(P<0.05)$ (Fig. 3g). These results demonstrate that TG2 mediates stress-induced depressive-like behaviors and decreases in TrkB levels in mice.

\section{Overexpression of TrkB in the prefrontal cortex of TG2-overexpressed mice ameliorates their depressive-like behaviors}

If the reduced levels of TrkB in the PFC of TG2 mice mediate the depressive-like phenotype, overexpression of TrkB should ameliorate the behavioral signs. We performed stereotaxic injection of lentiviral TrkB into the PFC of WT and TG2 mice (Fig. 4a). TrkB expression was confirmed by immunofluorescence and immunohistochemistry (Fig. 4b). Also, we found increased levels of TrkB in the PFC samples from lentiviral TrkB-injected mice as compared to lentiviral-GFP injected control mice $(P<0.05)$ (Fig. $4 \mathrm{c})$. In the behavioral tests, the hyperactivity (i.e., increase in distance traveled in the open field test) of TG2 mice was significantly reduced following TrkB overexpression into the PFC $(P<0.05)$ (Fig. 4d). In addition, lentiviral TrkB overexpression significantly reduced the immobility of TG2 mice in TST $(P<0.05)$ and FST $(P<0.05)($ Fig. $4 \mathrm{~d})$. These results strongly suggest that reduced TrkB signaling is involved in the depressive-like behaviors observed in TG2 mice. It is known that Trk receptors are recycled to the plasma membrane via recycling endosomes or transported from early to late endosomes and eventually to lysosomes for degradation. Since we found a significant reduction in TrkB protein levels in TG2 mice, we next examined whether the degradation of TrkB is higher when TG2 is overexpressed in primary cortical neurons with lentiviral particles. The halflife of TrkB was examined following BDNF treatment by measuring the ratios of TrkB at different time intervals to TrkB levels before BDNF treatment. Our data indicated that TrkB is more rapidly degraded in TG2 overexpressed neurons (Supplementary Fig. 9a). To track the intracellular localization of TrkB receptors, co-immunofluorescence was performed with the lysosomal marker, LAMP1. Under BDNF induction, TrkB was more localized to LAMP1 in the TG2 overexpressing neurons compared to the neurons treated with control lentiviral particles suggesting that LAMP1-vesicles initiate the lysosomal degradation (Supplementary Fig. 9b).

\section{Increases in TG2 mRNA and protein levels are detected in the prefrontal cortex of depressed suicide subjects}

To determine the importance of TG2 in depressive disorders in humans, we examined postmortem brain samples of depressed suicide subjects and nonsuicide controls. The postmortem samples comprised 28 men, half of whom were suicide completers who had been diagnosed with major depressive disorder. None of the evaluated covariates had a significant association with TG2 mRNA. RNA integrity $(r=0.248, p=0.20)$ and medication use $(r=0.303, p=0.12)$ had small associations with TG2 mRNA so these covariates were included in the ANCOVA model (Supplementary Table 1 and Table 2). When entered as covariates, neither RNA integrity $\left[F(1,24)=1.75, p=0.20, \eta_{p}^{2}=0.07\right]$ nor medication use 
$\left[F(1,24)=1.99, p=0.17, \eta_{\mathrm{p}}^{2}=0.08\right]$ had significant associations with TG2 mRNA and were subsequently removed from the model. The predicted main effect of affection status on TG2 mRNA was significant $[F(1,26)=4.59 p=0.042$, Cohen's $d=0.81]$. Overall, the depressed suicide subjects demonstrated greater TG2 mRNA than the control subjects (Supplementary Fig. 10a). Also, we found a significant increase in TG2 protein levels in the PFC of depressed suicide subjects as compared to control subjects (Supplementary Fig. 10b, c). In addition, a significant reduction in Rac1 protein levels (Supplementary Fig. 10b, d) was observed in the depressed suicide subjects.

\section{Discussion}

Collectively, the data we have presented in this report clearly suggest that TG2 is involved in depressive-like behaviors. TG2 has been shown to transamidate serotonin to Rac1, leading to the transient activation and proteasomal degradation of $\mathrm{Rac}^{2}$. We found a significant reduction in Rac1 levels in the PFC of TG2 overexpressed mice. Rac1 plays an important role in neuronal development, axon guidance, dendritic spine morphogenesis and maintenance, and in learning and memory processes ${ }^{10,11}$. Moreover, chronic stress has been shown to reduce Rac1 expression in rodents, and a significant reduction in Rac1 expression has been observed in the nucleus accumbens of depressed subjects ${ }^{12}$. Our data show a significant reduction in Rac1 expression in the PFC of depressed subjects, further suggesting that this GTPase plays an important role in the pathophysiology of depression. Furthermore, Rac1 has been shown to mediate the endocytosis of TrkB in neurons ${ }^{8}$. Interestingly, the behavioral deficits in TG2 overexpressed mice were ameliorated by overexpression of TrkB in the PFC and by antidepressant drug that can increase TrkB signaling, suggesting that impaired TrkB signaling might contribute to the depressive-like behaviors observed in TG2 mice.

Despite the above evidence that TG2 plays a pivotal role in depression, questions still remain as to how stress induces increases in TG2 expression. TG2 is a direct transcriptional target of NF- $\kappa$ B and the TG2 gene contains two consensus NF- $\kappa$ B binding sites ${ }^{13}$. Activation of NF- $\kappa \mathrm{B}$ has been shown to induce TG2 expression, and the NF- $\kappa \mathrm{B}$ subunit RelA is both necessary and sufficient to drive TG2 expression ${ }^{14}$. Chronic stress and/or inflammation are known to activate NF- $\mathrm{\kappa B}$ signaling and this signaling pathway is required for the depressive-like behavior in rodents caused by exposure to chronic stress ${ }^{15}$.

In the present study, we evaluated depressive-like behaviors in mice using the TST and FST. Although the FST was originally developed as a model for assessing antidepressant-like activity in rats ${ }^{16,17}$, a number of recent studies have employed this test for examining stressinduced depressive-like behavior in mice ${ }^{18}$. While the FST is sensitive to all of the effective antidepressant drug classes (and is currently the most widely used model for evaluating potential antidepressant drugs), its validity as a true measure of depression-like behavior and antidepressant activity of drugs has been debated due to potential confounding effects of altered locomotor activity ${ }^{19,20}$. Therefore, we performed locomotor activity assessments (open field test) along with the FST in our studies to determine whether the changes in immobility in the FST might be related to alterations in the general activity of the mice. It has been suggested that an increase in active behaviors in the FST coupled with a reduction 
in locomotor activity following acute antidepressant treatment validates the behavior in $\mathrm{FST}^{21}$. We found similar changes in the open field test and FST in TG2 mice following fluoxetine treatment. Although we observed significant changes in depressive-like behavior following acute fluoxetine treatment in mice, chronic administration of antidepressants is generally required to achieve a positive effect (with a delayed onset of action) on the Hamilton Depression Rating Scale (HAM-D) score in humans. The acute activation of TrkB in cortex after antidepressant administration is expected to lead to plastic changes in synaptic function and neuronal connectivity since TrkB signaling is known to stimulate outgrowth and regeneration of dendrites and axons ${ }^{22-24}$ and to promote synaptogenesis ${ }^{24}$. It is possible that posttranslational modifications of proteins involved in neuronal functions with acute antidepressant treatment results in changes in some behaviors and symptoms, whereas changes at the gene level following chronic administration underlies the more lasting therapeutic and prophylactic properties of antidepressants (i.e., effects associated with neurogenesis and long-tern changes in neuroplasticity). Such a slow development of neuronal changes might also explain in part the delayed onset of action of antidepressants in humans.

It is also important to note that the phenotypic alterations induced by the overexpression of TG2 in mice appeared to be relatively selective for depression and anhedonia-related behaviors as opposed to anxiety, schizophrenia-related signs. This argument is supported by the observation that (other than hyperactivity observed in the locomotor assessments) few notable effects were observed in the anxiety-related tests (elevated plus maze and light-dark box), sensorimotor gating task (PPI), or working/short-term memory task (Y-maze spontaneous alternation).

In conclusion, in this manuscript we have presented multiple lines of evidence (i.e., from neuronal culture experiments, behavioral and molecular studies in transgenic mice, and analyses of post mortem samples from depressed subjects) to support the argument that the transglutaminase enzyme TG2 contributes to the pathophysiology and symptoms of depression. Moreover, the reversal of chronic stress-induced behavioral deficits by cysteamine (a pharmacological inhibitor of TG2) in mice suggests that TG2 may represent a novel therapeutic target in depression.

\section{Materials and Methods}

\section{Animals}

Adult as well as timed pregnant $\mathrm{C} 57 \mathrm{BL} / 6 \mathrm{~J}$ mice were purchased from Charles River Laboratories (Wilmington, MA, USA). Transglutaminase 2 overexpressed mice (C57BL/6J) were provided by Janusz Tucholski, Department of Psychiatry and Behavioral Neurobiology, University of Alabama at Birmingham, $\mathrm{USA}^{7}$ and the colony was maintained in our animal housing facility at Augusta University. Mice were housed in groups of 4 mice in standard polypropylene cages in 12-h light-dark cycle in compliance with the US National Institute of Health guidelines and approved by Augusta University animal welfare guidelines. 8-10 week old male mice were used for the in vivo experiments. Mice were assigned to experimental groups based on their genotype. Selection of animal for lentiviral or pharmacological treatment was performed randomly and in a blinded manner. 


\section{Cell culture}

Primary cortical neurons were prepared at E16. Neurons were cultured in Neurobasal medium containing, B27 supplement, 10\% Fetal Bovine Serum (FBS), penicillin/ streptomycin mixture of antibiotics and Glutamax for 5 days. Media were changed with fresh media prior to the experimental treatment. Treatments were performed in neurons at day in vitro (DIV) 5-7.

\section{Drugs}

NSC 23766 (NSC, Tocris) and Fluoxetine (Sigma, St Louis, MO, USA) were administrated intraperitoneally (i.p; $100 \mu \mathrm{L}$ ) at a concentration of $2.5 \mathrm{mg}$ per kg body weight per day (mg $\mathrm{kg}^{-1} \mathrm{day}^{-1}$ ) and $20 \mathrm{mg} \mathrm{kg}^{-1}$ day $^{-1}$ respectively. Cysteamine (Sigma) was administrated at a concentration of $150 \mathrm{mg} \mathrm{kg}^{-1} \mathrm{day}^{-1}$ through drinking water for 21 days. Corticosterone (4pregnen-11b-diol-3 20-dione 21-hemisuccinate; Sigma) was dissolved in vehicle $(0.45 \%$ hydroxypropyl- $\beta$-cyclodextrin, Sigma). Corticosterone ( $35 \mu \mathrm{g} / \mathrm{mL}$, equivalent to $5 \mathrm{mg} \mathrm{kg}^{-1}$ day $^{-1}$ ) was delivered ad libitum in the drinking water. The dose and duration of corticosterone treatment in mice were selected based on earlier studies ${ }^{9,25}$, where the above dose and duration of treatment with CORT induced anxiety and depressive-like behaviors in mice. The fluid intake was monitored throughout the treatment period and the corticosterone dose was adjusted accordingly. Primary cortical neurons were treated with serotonin (5-HT; Sigma) at a concentration of 5-100 $\mu \mathrm{M}$ at DIV 5-7. In glucocorticoid receptor inhibitor studies, cells were treated with RU486 (1 $\mu \mathrm{M}$; Sigma) 30 min before 48-h corticosterone (1 $\mu \mathrm{M}$; Sigma) treatment. Neurons were treated with BDNF (prospec) at $100 \mathrm{ng} / \mathrm{ml}$ for indicated time period.

\section{Mouse genotyping}

$\mathrm{TG}^{+/+}$mice were bred on C57BL/6J backgrounds and their offspring were genotyped at by PCR of ear punch DNA (DNeasy kits; Qiagen). PCR reaction was performed over 35 cycles using GoTaq ${ }^{\circledR}$ Green Master Mix (Promega). Primers utilized were as follows: (i) PrP: sense primer $5^{\prime}$-GCTGAAGCATTCTGCCTTCCTAGTG-3' ${ }^{\prime}$ and an antisense primer 5'GTGGATACCCCCTCCCCCAGCCTAGACC $3^{\prime}$. (ii) htTG: sense primer 5'CTCGTGGAGCCAGTTAT CAACAG3' and antisense primer 5'TGAGGACATACTCCTGCCGCTCCTCTTCCGAGG3'. Annealing temperature was set at $60^{\circ} \mathrm{C}$. The products of the PCR reaction were visualized using agarose gel electrophoresis.

\section{Stereotaxic Injection of Lentivirus}

DNA for pCAG-trkB-EF1a-copGFP (p289) and the matching control, pCDH CAG eGFP EF1a copGFP (p375) were kindly provided by Mark H. Tuszynski (University of California, San Diego), and lentiviral particles were synthesized by Applied Biological Materials Inc. pLenti-GIII-CMV-mTGM2-GFP-2A-Puro, pLenti-CMV-GFP-2A-Puro-Blank Vector, Lentiviral vector expressing TG2 siRNA and Scrambled siRNA GFP were purchased from Applied Biological Materials Inc. Lentiviral particles were injected sterotaxically into mouse PFC. In brief, mice were received Buprenorphine $(0.05 \mathrm{mg} / \mathrm{kg}$, s.c.) for $15 \mathrm{~min}$ and then anesthetized with 1.5-2.5\% isoflourane mixed with oxygen in an induction chamber. Mice were maintained under deep anesthesia and mounted in a stereotaxic apparatus. Around $\sim 1$ 
$\mathrm{mm}$ size burr hole were made to perform a very small craniotomy on the right side as per the recommended coordinates ${ }^{26}$ (x .5 mm (lateral), y $1.0 \mathrm{~mm}$ (anteriorposterior A-P), with respect to bregma at 0 ), z $1.0 \mathrm{~mm}$ (dorso-ventral $\mathrm{D}-\mathrm{V}$ with brain surface at 0 ). A 30-gauge needle attached to a micro syringe containing lentvirus were inserted and lowered into the prefrontal cortical region of the brain. The microsyringe was left in place 5 min after each injection, and a total volume of $1.0 \mu \mathrm{l}\left(1 \times 10^{9}\right.$ infectious particles per milliliter $)$ of lentivirus was administered at a rate of $0.2 \mu \mathrm{l} / \mathrm{min}$ at each site (Stoelting Co). Mice will be allowed to recover from anesthesia, transferred to a clean cage with free access to chow diet and water. After the $2 \mathrm{wk}$ of injection, the injection site was confirmed by Ex vivo imaging analysis for the fluorescence of lentiviral particles.

\section{Chronic unpredictable stress (CUS) procedure}

CUS experiments were performed according to previously published procedure with modifications ${ }^{27}$. Three weeks prior to the beginning of the CUS paradigm, all animals were individually housed while all other aspects of housing remained as standard housing conditions. The details of each stressor, duration of stress exposure, and sequence of exposure is shown in Supplementary Table 3. The stress exposure regimen was mixed with the qualifier that exposures to the same stressor never occurred consecutively within a day. The controls received the same temporal and spatial sequence of habituation and test days that the stressed animals experienced.

\section{Sucrose preference test}

Mice were habituated to $1 \%$ sucrose solution for 3 days at the start of the experiment, in which two bottles of $1 \%$ sucrose solution were placed in each cage. After adaptation, mice were deprived of fluid for $16 \mathrm{~h}$. Mice were then presented with two identical bottles of $1 \%$ sucrose and water for $24 \mathrm{~h}$. The position of the bottles containing water or sucrose solution was switched halfway through the test. At the end of the 24-h test, sucrose and water consumption (ml) were measured, and the preference for sucrose was calculated as a percentage of consumed sucrose solution of the total amount of liquid drunk.

\section{Forced swim test}

Mice were subjected to one 5-min session of swimming in water. On the test day, mice were placed in a clear cylinder with water $\left(24^{\circ} \mathrm{C} \pm 1{ }^{\circ} \mathrm{C}, 45\right.$-cm depth). The sessions were recorded from the side, and the immobility time was scored by a blind observer. Immobility was calculated as the least amount of movement of experimental mice to stay afloat.

\section{Tail Suspension test}

Each mouse was suspended with a tape $0.75 \mathrm{~cm}$ away from their tails and elevated $70 \mathrm{~cm}$ above the floor. Behavior was recorded for $6 \mathrm{~min}$. Observer blinded to the subject's treatment group scored video recordings for time spent struggling or immobile.

\section{Y maze test}

The Y-maze was opaque white-cast acrylic with 3 arms (each $=30 \mathrm{~cm} \mathrm{~L} \times 8 \mathrm{~cm} \mathrm{~W} \times 6 \mathrm{~cm}$ $\mathrm{H})$ joined at one end to form a ' $\mathrm{Y}$ '-shape with a common central zone. Each trial was 
initiated by releasing a mouse into the center zone for a 10-minute free exploration period. Extra-maze, but not intra-maze, cues were visible to use as spatial landmarks. The apparatus was cleaned with $50 \%$ ethanol between trials. All trials were video-recorded (30 frames/ second, Basler GigE ace camera) to manually score the sequence that each mouse entered arms (in 1-minute bins) over the testing period. Subsequently, the first two trials were omitted and the total number of alternations (entries into three different arms consecutively) and arm entries were counted. The percent alternation was calculated, as follows: [(number of alternations)/(total possible alternations) $] \times 100 \%$.

\section{Open field test}

Mice activity in an open field was measured over a $10-$ min period $^{28}$. Time spent (sec.) in margin and center, total distance $(\mathrm{cm})$ and mean speed $(\mathrm{cm} / \mathrm{sec}$.) were measured during the session. Ethovision XT 10 (Noldus Information Technologies Inc, USA) software and open field chamber $(40 \times 40 \times 40 \mathrm{~cm})$, and a video camera was fixed over the chamber by an adjacent rod, an activity monitor, a programmer and a printer were used for analysis. Sessions total for all parameters were taken. Observations were recorded for $10 \mathrm{~min}$.

\section{Prepulse inhibition (PPI) test}

On the first day, mice were allowed to habituate to the testing chamber apparatus (San Diego Instruments, San Diego, CA) for 20 minutes. The second day consisted of the mice being exposed to various decibels $(\mathrm{dB})$ of auditory stimuli, randomized for a total of 24 trials to acclimate the mice to the startle and prepulse stimuli. The third day the mice underwent testing for a total of 60 psuedorandomized trials. These trials were set up such that no prepulse amplitude repeated itself consecutively or have the three prepulse stimuli in consecutive order (i.e. $75,80,85 \mathrm{~dB}$ ). A continuous background noise of $70 \mathrm{~dB}$ was used. The nulstim background noise was $70 \mathrm{~dB}$ with duration of $40 \mathrm{~ms}$. Pre-pulse values were 75 , 80 , and $85 \mathrm{~dB}$ at $20 \mathrm{~ms}$ durations. Startle amplitude of $120 \mathrm{~dB}$ at $40 \mathrm{~ms}$ was used. The interstimulus interval between the prepulse/startle presentations ranged from 10 to 30 secs and the total experimental session lasted about $23 \mathrm{~min}$.

\section{Light-Dark preference test}

In this test, the mouse open field activity monitors were fitted with dark box inserts (which are opaque to visible light) to cover one-half of the open field area thus separating the apparatus into two zones of equal area (i.e., a brightly lit zone and a darkened zone). Desk lamps located above the activity monitors were used to provide an illumination level of approximately 1000 lux in the brightly lit zone, whereas the illumination level in the darkened zone was approximately 5 lux. The time spent and distance traveled in the light and dark zones of the apparatus were recorded for the 10 min test session.

\section{Elevated plus maze}

The elevated plus maze consisted of two orthogonal closed $(15 \mathrm{~cm} \times 6 \mathrm{~cm} \times 30 \mathrm{~cm})$ and open $(1 \mathrm{~cm} \times 6 \mathrm{~cm} \times 30 \mathrm{~cm})$ arms forming a cross, with a quadrangular $(6 \mathrm{~cm} \times 6 \mathrm{~cm})$ area located at the intersection. The maze was placed $50 \mathrm{~cm}$ above the floor and was made of black plastic. The degree of anxiety was assessed by measuring the time spent on the open and closed 
arms and the number of entries made into each arm. Each test lasted 10 min, and was initiated by placing a mouse on the center facing an open arm. The behavior of the animal was recorded with a video camera and later scored by an independent observer blind to treatment. The following behaviors were scored and compared between groups: open and closed arm entries (defined as all 4 paws passing the threshold between the center/ intersection portion of the maze and the beginning a particular arm), time spent and distance traveled in the closed and open arms as well as in the center area.

\section{Western blotting}

Animals were sacrificed by cervical dislocation, and PFC, hippocampus and striatum samples were collected according to a mouse brain atlas ${ }^{29}$. Brain tissues and primary cortical neuronal cells were lysed in ice-cold lysis buffer ${ }^{30}$. Protein was electrophoretically separated on a SDS PAGE gel and transferred to a nitrocellulose membrane. Blots were incubated in the appropriate primary antibody specific for TG2 (Cell Signaling 3557; 1:1,000); TrkB (Cell Signaling 4603; 1:1,000); p-TrkB (1:1000, kindly gifted by Dr Chao, New York University School of Medicine) ${ }^{31}$; p-ERK (Thr202/Tyr204; Cell Signaling 4370; 1:1,000); ERK (Cell Signaling 9102; 1:1,000); p-Akt (Ser473; Cell Signaling 9018; 1:1,000); Akt (Cell Signaling 9272; 1:1,000); $\beta$-Actin (Sigma A4700; 1:20,000); Rac1 (Santa Cruz Biotech SC-95; 1:500) and developed with the ECL Plus Western Blotting Detection System (GE Healthcare). Optical densities of the bands were analyzed using ImageJ software (NIH). For analysis, protein levels were normalized to $\beta$-actin levels then expressed as a fold change of that in control animals. For figure panels, contrasts have been adjusted linearly for easier viewing of bands.

\section{Quantitative reverse transcriptase PCR (qRT-PCR)}

RNA was purified using a commercially available kit (SV RNA Isolation, Promega, Madison, WI, USA), qRT-PCR was performed on a MasterCycler (Eppendorf) using a SuperScript III Platinum SYBR Green One-Step qRT-PCR kit (Invitrogen, Carlsbad, CA, USA). Primers for TG2 were synthesized by Integrated DNA Technologies. Primers used were, forward: $5^{\prime}$-tcaactgcaacgatgaccagg- $3^{\prime}$ and reverse: $5^{\prime}$-tgttctggtcatgggccg- $3^{\prime}$. Ct values of TG2 were normalized to that of housekeeping gene ( $\beta$-Actin).

\section{Immunofluorescence staining of cultured neurons}

For immunofluorescence staining of primary cortical neurons, cultured neurons were transfected with control or TG2 lentiviral particles using Effectene Transfection Reagent (Qiagen) $48 \mathrm{~h}$ before BDNF (100 ng/mL) treatment. Cells were washed and permeabilized for $5 \mathrm{~min}$ with $0.2 \% \mathrm{v} / \mathrm{v}$ triton-X100 and blocked for $1 \mathrm{hr}$ at room temperature with $5 \%$ normal donkey serum. Anti-TrkB (abcam ab52191; 1:1000) and anti-Lamp1 (DSHB (1D4B); 1:500) staining was performed overnight at $4^{\circ} \mathrm{C}$ followed by rinsing with PBS and incubation for $1 \mathrm{hr}$ at room temperature with $\mathrm{Cy} 2$ and $\mathrm{Cy} 3$ conjugated secondary antibodies (Jackson Immunoresearch). Cells were then washed and mounted using ProLong® Gold Antifade Mountant containing DAPI (Molecular Probes). Confocal images were obtained using a Zeiss LSM 510 META confocal microscope. Pictures were taken under a 40× PlanApochromat objective. 


\section{High-performance liquid chromatography (HPLC)}

Trunk blood samples were collected into microcentrifuge tubes containing ACD anticoagulant solution A (BD). Biogenic amines in trunk blood and brain samples were determined by a specific HPLC assay using an Decade (oxidation, 0.7) electrochemical detector (Antec). Biogenic amines were eluted with a mobile phase consisting of $89.5 \%$ 0.1M TCA, 10-2 M sodium acetate, 10-4 M EDTA, and 10.5\% (vol/vol) methanol (pH 3.8). Concentration was determined by comparison with injections of known standards.

\section{Immunohistochemistry}

Mice were anesthetized and transcardially perfused with phosphate-buffered saline (PBS) followed by $4 \%$ paraformaldehyde in PBS. Brains were removed and PFC cryostat sections (10 mm thick, coronal sections) were prepared with standard protocols. For immunostaining, the brain section was reacted with Anti-TG2 (abcam ab421; 1:300) and anti-PV (Swant 235; 1:500) or anti-CAMKII (BD Transduction Lab. 611292; 1:500) followed by Cy2 and Cy3 conjugated secondary antibodies. Sections were mount with Antifade Mountant containing DAPI and images were taken using confocal microscope under $40 \times$ objective. $3,3^{\prime}$ Diaminobenzidine (DAB) staining was carried out using the ABC Kit, followed by the Elite DAB Staining Kit, according to the manufacturer's instructions (Vector Laboratories).

\section{Spine analysis}

Golgi staining was carried out using the FD Rapid Golgi Stain Kit (FD Neurotechnologies, Baltimore, $\mathrm{MD})^{32}$. In brief, the mouse brains were placed in an appropriate impregnation solution and stored in the dark for 2 weeks at room temperature. Next, the brains were transferred to a second impregnation solution and incubated for 3 days at $4^{\circ} \mathrm{C}$. Finally, semihorizontal sections $(120 \mu \mathrm{m})$ were prepared with an oscillating tissue slicer (Leica, VT1000, Wetzlar, Germany). Sections were stained and then mounted on gelatin coated slides. Slides were then rinsed again in distilled water and dehydrated in absolute alcohol, cleared with xylene, mounted on slides and covered with nonacidic synthetic balsam and coverslips.

\section{Postmortem samples}

Human postmortem brain tissue samples (Brodmann's area 10) from suicide completers $(\mathrm{N}=14)$ and psychiatrically normal controls $(\mathrm{N}=14)$ were used in the current study. The samples were obtained from the Quebec Suicide Brain Bank (QSBB; Douglas Institute; www.douglas.qc.ca/suicide). The study was granted by the Douglas Hospital Institutional Review Board and, written informed consent was obtained from each participating family (Declaration of Helsinki, 1964). A description of the QSBB and other detailed demographics and samples' quality were published earlier ${ }^{33,34}$.

\section{Statistical Analysis}

No statistical methods were used to predetermine sample sizes in mouse studies, but our sample sizes are similar to those reported in previous publications ${ }^{9,15,25}$. For mouse studies, data were analyzed using two-tailed Student's t-tests (for two-group comparisons) or Analysis of Variance (ANOVA; for multiple-group comparisons). $\mathrm{p}<0.05$ was considered significant. Post hoc analyses were carried out using Bonferroni's test. For postmortem 
studies, Analysis of Covariance (ANCOVA) was used to examine differences in TG2 mRNA between postmortem samples of depressed suicide subjects and the controls (Affection Status). Group differences in TG2 mRNA were examined with age, post mortem interval (PMI), pH, RNA integrity, substance use at death, and psychiatric medication use evaluated for inclusion as possible covariates. Only covariates with at least small associationscorrelations of 0.20 and higher-with TG2 were considered for inclusion in the ANCOVA model. Candidate covariates that were significantly correlated with TG2 mRNA were included in the model. Exact probability $(p)$ values of less than 5\% were flagged as statistically significant. Cohen $d$ was computed as a measure of effect size difference. All analyses were performed using SPSS Statistics 20 software (IBM).

\section{Supplementary Material}

Refer to Web version on PubMed Central for supplementary material.

\section{Acknowledgments}

We thank Dr. Chao (New York University School of Medicine) for the phospho-TrkB antibody. The authors would also like to acknowledge Quebec Suicide Brain Bank for postmortem tissue samples, Mark H. Tuszynski

(University of California, San Diego) for the TrkB and control vectors, Edmond R Hollis (University of California, San Diego) for his helpful comments on the lentiviral experiments, Janusz Tucholski (University of Alabama) for TG2 mice, Vanderbilt KC/VBI Neurochemistry Core Lab (Vanderbilt University) for HPLC analysis, and Augusta University Small Animal Behavior core for the behavior experiments. This research was supported by US National Institute of Mental Health grant R01 MH 097060 (A.P.).

\section{References}

1. Martinowich K, Lu B. Interaction between BDNF and serotonin: role in mood disorders. Neuropsychopharmacology : official publication of the American College of Neuropsychopharmacology. 2008; 33:73-83. [PubMed: 17882234]

2. Dai Y, Dudek NL, Patel TB, Muma NA. Transglutaminase-catalyzed transamidation: a novel mechanism for Rac1 activation by 5-hydroxytryptamine2A receptor stimulation. The Journal of pharmacology and experimental therapeutics. 2008; 326:153-162. [PubMed: 18400843]

3. Guilluy C, Rolli-Derkinderen M, Tharaux PL, Melino G, Pacaud P, Loirand G. Transglutaminasedependent RhoA activation and depletion by serotonin in vascular smooth muscle cells. The Journal of biological chemistry. 2007; 282:2918-2928. [PubMed: 17142836]

4. Lorand L, Graham RM. Transglutaminases: crosslinking enzymes with pleiotropic functions. Nature reviews Molecular cell biology. 2003; 4:140-156. [PubMed: 12563291]

5. Milakovic T, Tucholski J, McCoy E, Johnson GV. Intracellular localization and activity state of tissue transglutaminase differentially impacts cell death. The Journal of biological chemistry. 2004; 279:8715-8722. [PubMed: 14670969]

6. Doye A, Mettouchi A, Bossis G, Clément R, Buisson-Touati C, Flatau G, et al. CNF1 exploits the ubiquitin-proteasome machinery to restrict Rho GTPase activation for bacterial host cell invasion. Cell. 2002; 111:553-564. [PubMed: 12437928]

7. Tucholski J, Roth KA, Johnson GV. Tissue transglutaminase overexpression in the brain potentiates calcium-induced hippocampal damage. Journal of neurochemistry. 2006; 97:582-594. [PubMed: 16539654]

8. Trovo L, Van Veldhoven PP, Martin MG, Dotti CG. Sphingomyelin upregulation in mature neurons contributes to TrkB activity by Rac1 endocytosis. Journal of cell science. 2011; 124:1308-1315. [PubMed: 21444756]

9. Kutiyanawalla A, Terry AV Jr, Pillai A. Cysteamine attenuates the decreases in TrkB protein levels and the anxiety/depression-like behaviors in mice induced by corticosterone treatment. PloS one. 2011; 6:e26153. [PubMed: 22039440] 
10. Koh CG. Rho GTPases and their regulators in neuronal functions and development. Neuro-Signals. 2006; 15:228-237. [PubMed: 17409776]

11. Martinez LA, Tejada-Simon MV. Pharmacological inactivation of the small GTPase Rac1 impairs long-term plasticity in the mouse hippocampus. Neuropharmacology. 2011; 61:305-312. [PubMed: 21569781]

12. Golden SA, Christoffel DJ, Heshmati M, Hodes GE, Magida J, Davis K, et al. Epigenetic regulation of RAC1 induces synaptic remodeling in stress disorders and depression. Nature medicine. 2013; 19:337-344.

13. Brown KD. Transglutaminase 2 and NF-kappaB: an odd couple that shapes breast cancer phenotype. Breast cancer research and treatment. 2013; 137:329-336. [PubMed: 23224146]

14. Ai L, Skehan RR, Saydi J, Lin T, Brown KD. Ataxia-Telangiectasia, Mutated (ATM)/Nuclear Factor kappa light chain enhancer of activated B cells (NFkB) signaling controls basal and DNA damage-induced transglutaminase 2 expression. The Journal of biological chemistry. 2012; 287:18330-18341. [PubMed: 22493284]

15. Koo JW, Russo SJ, Ferguson D, Nestler EJ, Duman RS. Nuclear factor-kappaB is a critical mediator of stress-impaired neurogenesis and depressive behavior. Proceedings of the National Academy of Sciences of the United States of America. 2010; 107:2669-2674. [PubMed: 20133768]

16. Porsolt RD, Le Pichon M, Jalfre M. Depression: a new animal model sensitive to antidepressant treatments. Nature. 1977; 266:730-722. [PubMed: 559941]

17. Porsolt RD, Anton G, Blavet N, Jalfre M. Behavioural despair in rats: a new model sensitive to antidepressant treatments. European Journal of Pharmacology. 1978; 47:379-391. [PubMed: 204499]

18. Bogdanova OV, Kanekar S, D'Anci KE, Renshaw PF. Factors influencing behavior in the forced swim test. Physiology \& Behavior. 2013; 118:227-239. [PubMed: 23685235]

19. Borsini F, Volterra G, Meli A. Does the behavioral "despair" test measure "despair"? Physiology \& Behavior. 1986; 38:385-6. [PubMed: 3786519]

20. Borsini F, Meli A. Is the forced swimming test a suitable model for revealing antidepressant activity? Psychopharmacology. 1988; 94:147-160. [PubMed: 3127840]

21. Slattery DA, Cryan JF. Using the rat forced swim test to assess antidepressant-like activity in rodents. Nature Protocols. 2012; 7:1009-1014. [PubMed: 22555240]

22. McAllister AK, Katz LC, Lo DC. Neurotrophins and synaptic plasticity. Annual Reviews in Neuroscience. 1999; 22:295-318.

23. Mamounas LA, Altar CA, Blue ME, Kaplan DR, Tessarollo L, Lyons WE. BDNF promotes the regenerative sprouting, but not survival, of injured serotonergic axons in the adult rat brain. Journal of Neuroscience. 2000; 20:771-782. [PubMed: 10632606]

24. Poo MM. Neurotrophins as synaptic modulators. Nature Reviews in Neuroscience. 2001; 2:24-32. [PubMed: 11253356]

25. David DJ, Samuels BA, Rainer Q, Wang JW, Marsteller D, Mendez I, et al. Neurogenesisdependent and -independent effects of fluoxetine in an animal model of anxiety/depression. Neuron. 2009; 62:479-493. [PubMed: 19477151]

26. Bearer EL, Zhang X, Janvelyan D, Boulat B, Jacobs RE. Reward circuitry is perturbed in the absence of the serotonin transporter. NeuroImage. 2009; 46:1091-1104. [PubMed: 19306930]

27. Miller LL, Ward SJ, Dykstra LA. Chronic unpredictable stress enhances cocaine-conditioned place preference in type 1 cannabinoid receptor knockout mice. Behavioural pharmacology. 2008; 19:575-581. [PubMed: 18690112]

28. Yamamoto M, Tamura A, Kirino T, Shimizu M, Sano K. Behavioral changes after focal cerebral ischemia by left middle cerebral artery occlusion in rats. Brain research. 1988; 452:323-328. [PubMed: 3401738]

29. Purger D, McNutt T, Achanta P, Quiñones-Hinojosa A, Wong J, Ford E. A histology-based atlas of the C57BL/6J mouse brain deformably registered to in vivo MRI for localized radiation and surgical targeting. Physics in medicine and biology. 2009; 54:7315-7327. [PubMed: 19926915] 
30. Pandya CD, Pillai A. TrkB interacts with ErbB4 and regulates NRG1-induced NR2B phosphorylation in cortical neurons before synaptogenesis. Cell communication and signaling : CCS. 2014; 12:47. [PubMed: 25052836]

31. Jeanneteau F, Garabedian MJ, Chao MV. Activation of Trk neurotrophin receptors by glucocorticoids provides a neuroprotective effect. Proceedings of the National Academy of Sciences of the United States of America. 2008; 105:4862-4867. [PubMed: 18347336]

32. Chakraborti A, Allen A, Allen B, Rosi S, Fike JR. Cranial irradiation alters dendritic spine density and morphology in the hippocampus. PloS one. 2012; 7:e40844. [PubMed: 22815839]

33. Freemantle E, Chen GG, Cruceanu C, Mechawar N, Turecki G. Analysis of oxysterols and cholesterol in prefrontal cortex of suicides. The international journal of neuropsychopharmacology / official scientific journal of the Collegium Internationale Neuropsychopharmacologicum. 2013; 16:1241-1249.

34. Freemantle E, Mechawar N, Turecki G. Cholesterol and phospholipids in frontal cortex and synaptosomes of suicide completers: relationship with endosomal lipid trafficking genes. Journal of psychiatric research. 2013; 47:272-279. [PubMed: 23164340] 
a
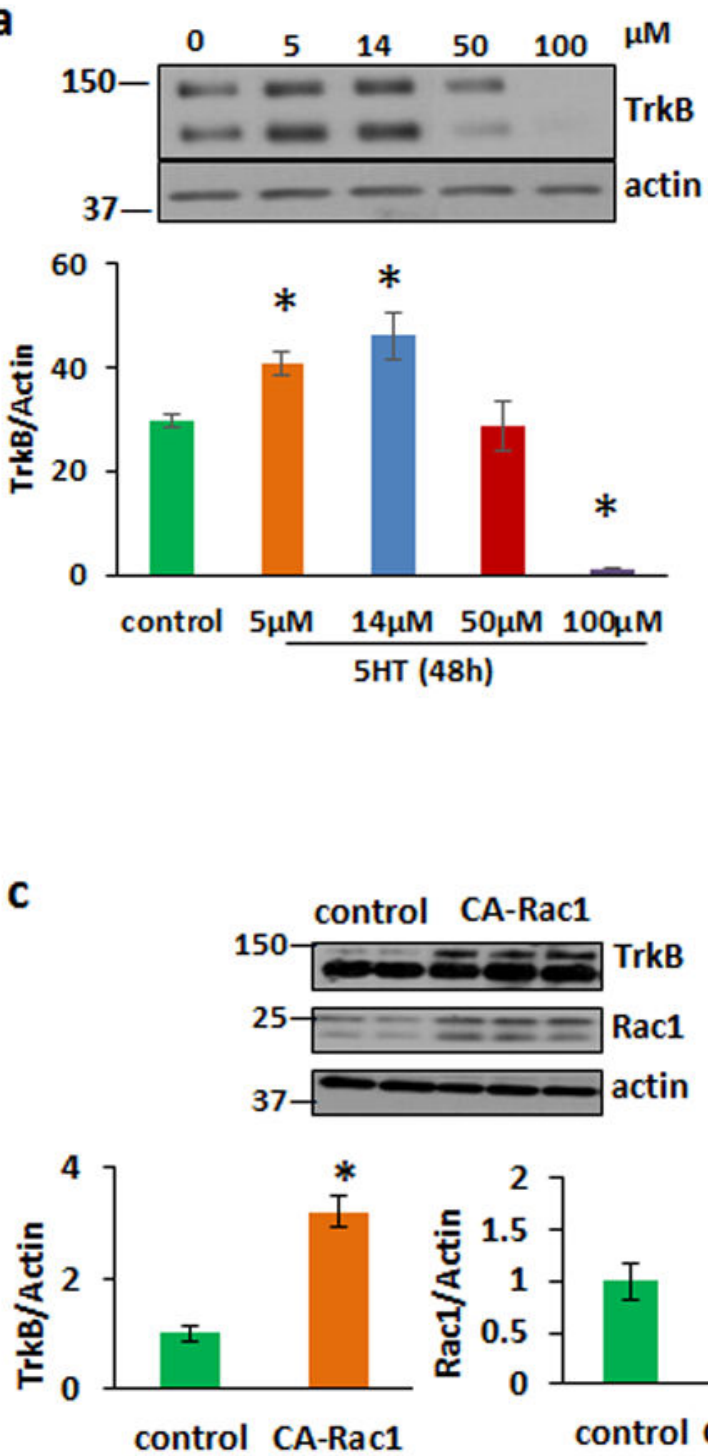

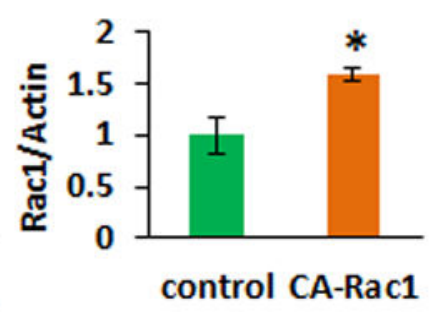

b
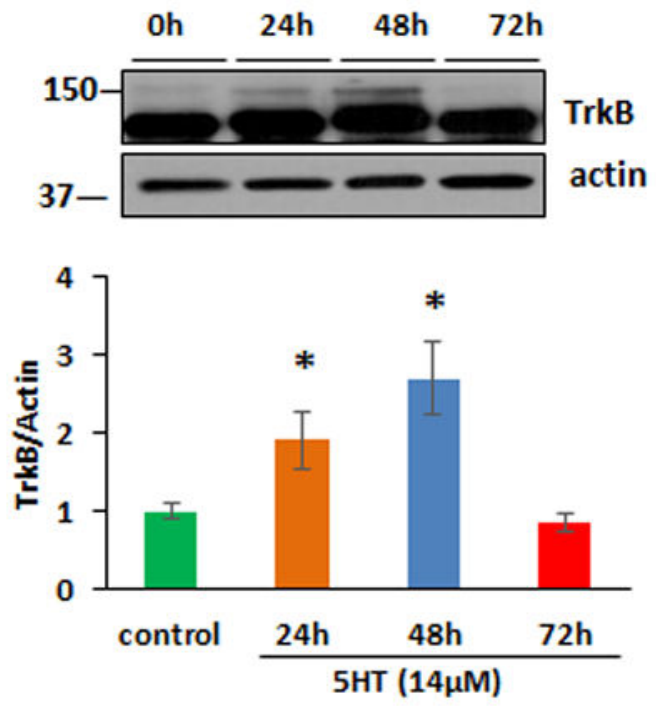

d
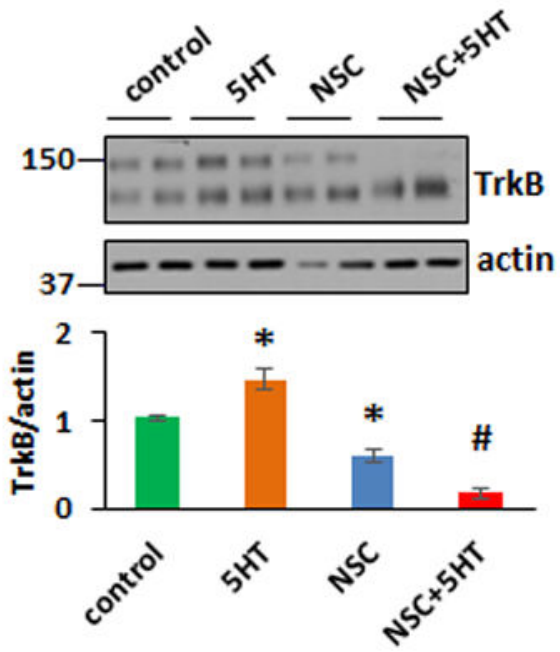

Figure 1. Rac1 mediates 5-HT-induced increase in TrkB levels in neurons Dose (a) and time (b) dependent effect of 5-HT treatment on TrkB protein levels in primary cortical neurons. (c) Rac1 and TrkB protein levels in primary cortical neurons transfected with empty or catalytically active (CA)-Rac1 constructs $(n=8)$. (d) Effect of Rac1 inhibitor NSC23766 (NSC; $200 \mu \mathrm{M}$ ) on 5-HT-induced increase in TrkB protein levels in primary cortical neurons $(\mathrm{N}=4-6)$. Data are expressed as mean \pm s.e.m. $* P<0.05$ vs control; ${ }^{\#} P<$ 0.01 vs 5-HT-treated neurons; one-way analysis of variance (ANOVA) (a,b,d); Student's $t$ test (c). 

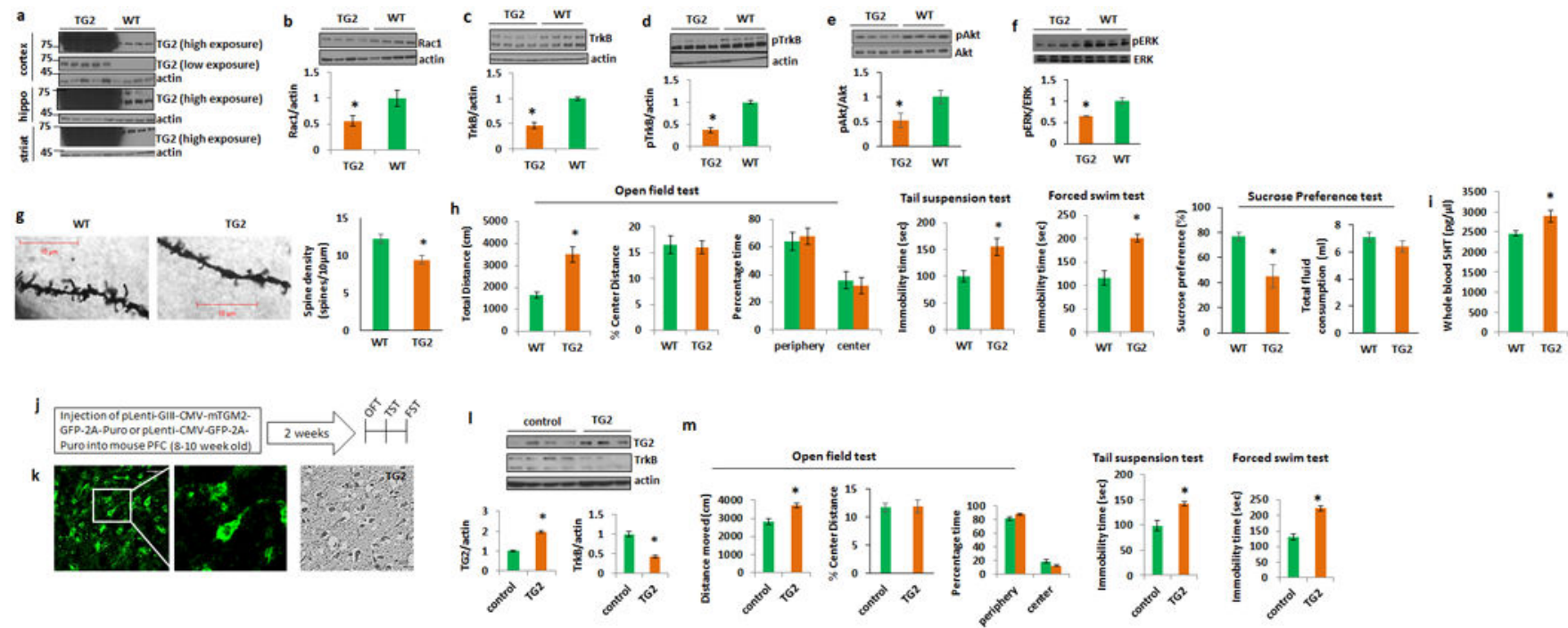

Figure 2. TG2 overexpression induces reductions in TrkB and Rac1 protein levels in PFC and depressive-like behavior in mice

(a) Representative immunoblot data showing TG2 expression in prefrontal cortex (cortex), hippocampus (hippo) and striatum (striat) of male TG2 overexpressed (TG2) and wild-type (WT) mice. (b) Rac1 and (c) TrkB protein levels in the prefrontal cortex (PFC) of TG2 $(n=6)$ and WT ( $n=6)$ mice. (d) pTrkB protein levels normalized to actin, (e) pAkt protein levels normalized to Akt, and (f) pERK protein levels normalized to ERK in the PFC of male TG2 ( $n=6)$ and WT ( $n=6)$ mice. (g) Left. Representative images of high-magnification $z$ stack projections of secondary branches of apical dendrites in the superficial layer and deep layers of PFC. Right. Spine density from male WT ( $\mathrm{n}=25$ branches from 6 cells; $n=3$ ) and TG2 ( $\mathrm{n}=27$ branches from 7 cells; $n=3$ ) mice. (h) Left. Distance traveled, \% distance in center, and time spent in the periphery and center area of the chamber in the open field test for WT ( $n=10)$ and TG2 $(n=10)$ mice. Center. The immobility time measured in tail suspension test and forced swim test for WT $(n=10)$ and TG2 $(n=10)$ mice. Right. Preference for sucrose and total fluid consumption over $24 \mathrm{~h}(\mathrm{ml})$ measured in sucrose preference test for WT $(n=10)$ and TG2 $(n=10)$ mice. (i) HPLC measurement of 5-HT in whole blood of WT $(n=6)$ and TG2 $(n=6)$ mice. (j) Schematic representation of experiment for lentiviral overexpression of TG2 into mouse PFC. (k) Left. Representative photographs of GFP-expressing neurons from the lentiviral TG2-injected mouse PFC. Right.

Representative image from 3,3'-Diaminobenzidine (DAB) staining showing TG2 expression in the injection site of lentiviral TG2-administered mouse PFC. (l) Top. Representative immunoblot data showing TG2, TrkB and $\beta$-actin expression in the PFC of mice injected with control or TG2 lentiviral particles. Bottom. TG2 and TrkB protein levels normalized to $\beta$-actin in the PFC of mice injected with control $(n=6)$ or TG2 (n=6) lentiviral particles. $(\mathbf{m})$ Left. Distance traveled, \% distance in center, and time spent in the periphery and center area of the chamber was calculated in the open field test for control $(n=8)$ or TG2-lentivirusinjected $(n=8)$ mice. The immobility time measured in (center) tail suspension test and (right) forced swim test for control $(n=8)$ or TG2 lentivirus-injected $(n=8)$ mice. Data are expressed as mean \pm s.e.m. $* P<0.05$ vs WT (b-f,h,i) or control lentiviral-treated mice $(\mathbf{l}, \mathbf{m})$; Student's $t$ test. 

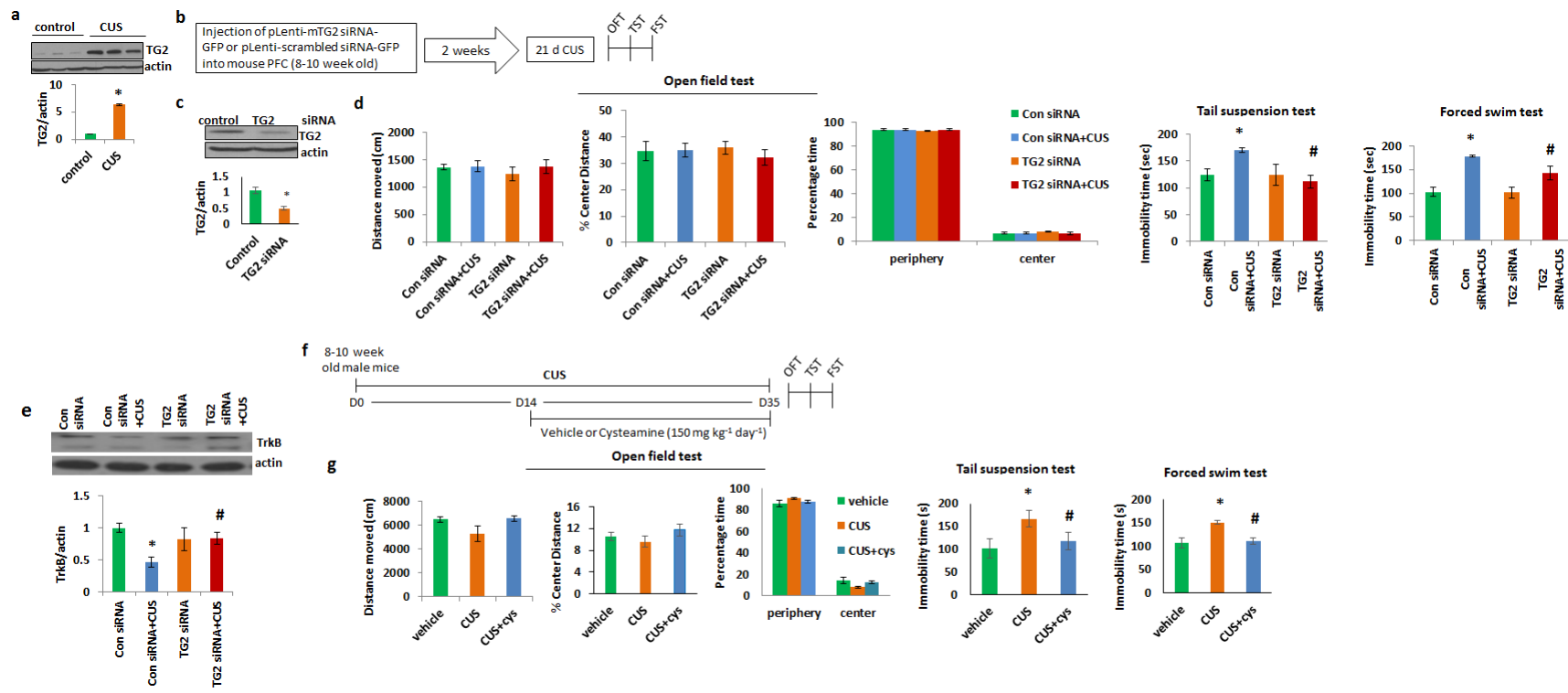

Figure 3. TG2 mediates the CUS-induced changes in depressive-like behavior and TrkB protein levels in mice

(a) Top. Representative immunoblot data showing TG2 and $\beta$-actin expression in the PFC of male mice exposed to CUS ( $n=6$ ) or no stress (control) ( $n=6)$. Bottom. TG2 protein levels normalized to $\beta$-actin. (b) Schematic representation of stereotaxic injection of control or TG2 siRNA lentiviral particles into mouse PFC cortex followed by chronic unpredictable stress (CUS) for 21 days. (c) Top. Representative immunoblot data showing TG2 and $\beta$-actin expression in the PFC of mice injected with control $(n=6)$ or TG2 (n=6) siRNA particles.

Bottom. TG2 protein levels normalized to $\beta$-actin. (d) Left. Distance traveled, \% distance in center, and time spent in the periphery and center area of the chamber was calculated in the open field test for control or TG2 siRNA-injected mice exposed to CUS or no stress ( $n=8$ per group). The immobility time measured in (center) tail suspension test and (right) forced swim test for control or TG2 siRNA-injected mice exposed to CUS or no stress ( $n=8$ per group). (e) Silencing TG2 levels by TG2 siRNA lentiviral particles attenuated CUS- induced decrease in TrkB protein levels in mouse PFC. Top. Representative immunoblot data showing TrkB and $\beta$-actin expression in the PFC of control $(n=6)$ or TG2 (n=6) siRNAinjected mice exposed to CUS or no stress. Bottom. TrkB protein levels normalized to $\beta$ actin. (f) Schematic representation of treatment plan for mice exposed to chronic unpredictable stress (CUS) for 5 weeks in presence or absence of cysteamine during the last three weeks of CUS exposure. (g) Left. Distance traveled, \% distance in center, and time spent in the periphery and center area of the chamber was calculated in the open field test for mice exposed to CUS in presence of absence of cysteamine ( $n=8$ per group). The immobility time measured in (center) tail suspension test and (right) forced swim test for mice exposed to CUS in presence of absence of cysteamine ( $n=8$ per group). Data are expressed as mean \pm s.e.m. ${ }^{*} P<0.05$ vs control (a,c-e) or vehicle $(\mathbf{g}) ;{ }^{\#} P<0.05$ vs consiRNA+CUS $(\mathbf{d}, \mathbf{e})$ or CUS (g); Student's $t$ test (a,c); two-way ANOVA (d,e); One-way ANOVA (g). 

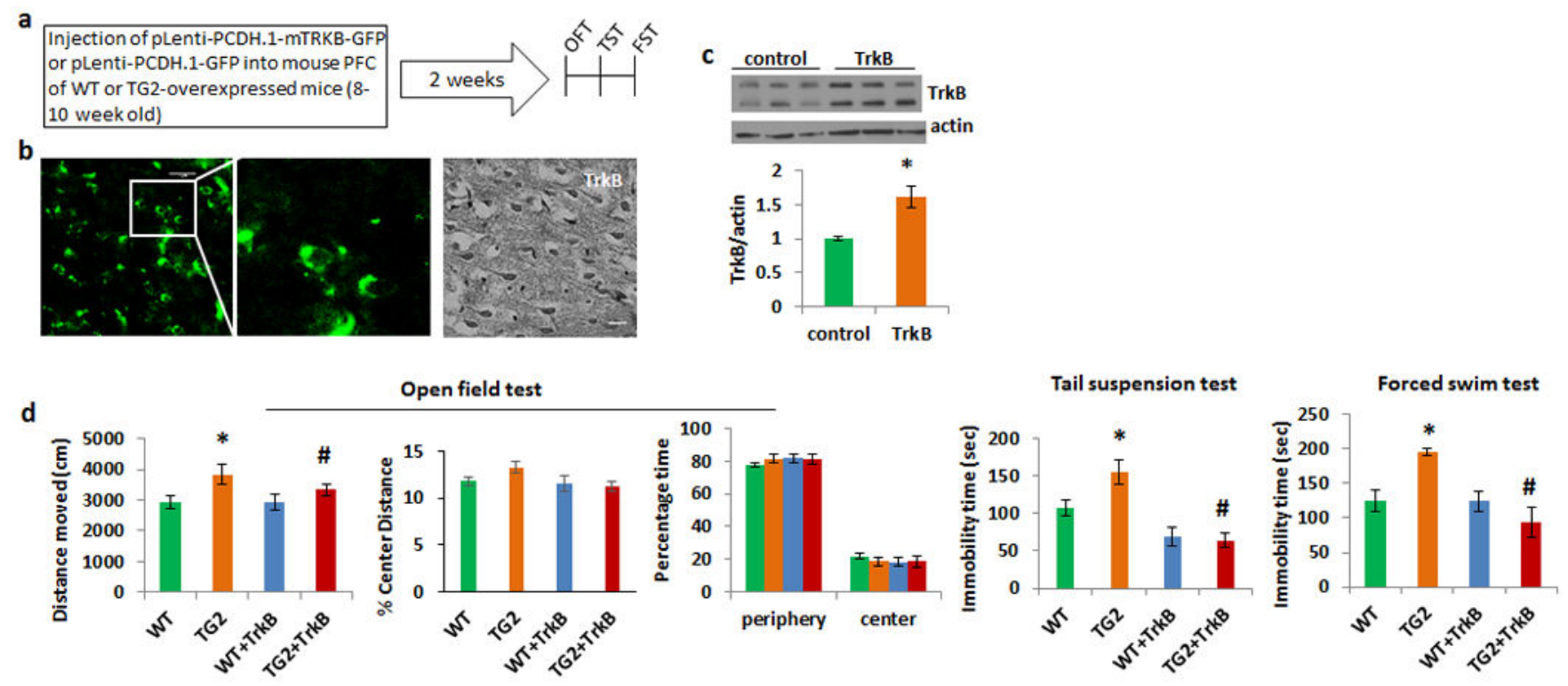

Figure 4. Overexpression of TrkB in the prefrontal cortex of TG2-overexpressed mice rescues their depressive-like behavior

(a) Schematic representation of experiment for lentiviral overexpression of TrkB into PFC of wildtype littermates (WT) or TG2-overexpressed (TG2) mice. (b) Left. Representative photographs of GFP-expressing neurons from the lentiviral TrkB -injected mouse PFC.

Right. Representative image from 3,3'-Diaminobenzidine (DAB) staining showing TrkB expression in the injection site of lentiviral TrkB-administered mouse PFC. (c) Top.

Representative immunoblot data showing TrkB and $\beta$-actin expression in the PFC of mice injected with control $(n=6)$ or TrkB $(n=6)$ lentiviral particles. Bottom. TrkB protein levels normalized to $\beta$-actin in the PFC of mice injected with control or TrkB lentiviral particles. (d) Left. Distance traveled, \% distance in center, and time spent in the periphery and center area of the chamber was calculated in the open field test for WT and TG2 mice injected with control or TrkB lentiviral particles ( $n=8$ per group). The immobility time measured in (center) tail suspension test and (right) forced swim test for WT and TG2 mice injected with control or TrkB lentiviral particles ( $n=8$ per group). Data are expressed as mean \pm s.e.m. $* P<$ 0.05 vs control lentiviral-treated WT mice. ${ }^{\#} P<0.05$ vs control lentiviral-treated TG2 mice; Student's $t$ test (c); two-way ANOVA (d). 